\title{
Surface Acoustic Wave Actuated Lab-on-Chip System for Single Cell Analysis
}

Stefan Thalhammer ${ }^{1 *}$ and Achim Wixforth ${ }^{2}$

${ }^{1}$ Helmholtz ZentrumMünchen, German Research Center for Environmental Health, Institute of Radiation Protection, IngolstädterLandstrasse 1, D-85764 Neuherberg, Germany

${ }^{2}$ Augsburg Center for Innovative Technologies ACIT, University of Augsburg, Experimental Physics I, D-86159 Augsburg, Germany

\begin{abstract}
Collection, selection, amplification and detection of minimal amounts of biological samples became part of everyday life in medical and biological laboratories. The goal of the lab-on-a-chip technology is to automate standard laboratory processes and to analyze smallest samples reproducibly and reliably in a miniaturized format. Until now, however, microfluidic devices are not yet standardized equipment found in biologists' toolboxes. Just one of the obstacles is the difficulty of producing them. We review various approaches for lab-on-chip systems reported in the literature but focus on our own system, based on surface acoustic wave (SAW) actuation. Finally, we present a freely programmable planar chip system actuated by this operating principle and show that it has several advantages over 'conventional' microfluidic channel systems. Apart from avoiding the notorious problems of clogging, large pressure drops, and large surface area, our approach minimizes the risk of contamination and loss of analyte. Entering pointof-care diagnosis, the necessity of single cell analysis is dramatically increasing. To optimize a tumor therapy, for example, knowledge of the genetic composition of the heterogenic tumor tissue is essential. Hence, there exists the need for a reliable analysis system, which can be easily adapted to altering problems without reduction in reliability and reproducibility.
\end{abstract}

Keywords: Lab-on-chip; Micro dissection; Microfluidics; Single cell analysis; Surface acoustic waves; VR-PCR; Low-pressure transfer

\section{Introduction}

Approximately two decades ago, a handful of researchers began discussing an intriguing idea: Could the equipment needed for everyday chemistry and biology procedures possibly be shrunk to fit on a chip the size of a fingernail? Miniature devices for, say, analyzing DNA and proteins should be faster and cheaper than conventional versions. Nowadays, the 'lab-on-a-chip'represents a quite mature and advanced technology that integrates a microfluidic system on a microscale device. Such a 'laboratory' is created by means of channels, mixers, reservoirs, diffusion chambers, integrated electrodes, pumps, valves and more. The ability to precisely control various parameters such as the choice of substrate, flow rate, buffer composition and surface chemistry in these micro scale devices, makes them ideal for a broad spectrum of cell-biology-based applications. These range from high throughput screening of single cells [1] and 3-D scaffolds for tissue engineering [2] to complex biochemical assays like polymerase chain reaction PCR (for a comprehensive review, see [3]), point of care diagnosis [4] and drug detection [5]. While the industrial approach to complexity has been to develop elaborate mechanical high throughput workstations, the technology comes at a price, requiring considerable expense, space and labor in the form of operator training and maintenance. For small laboratories or research institutions, this technology is basically out of reach. Also, many of the existing solutions are deployable for very specific purposes or assays, only. Devices consisting of addressable, maybe programmable micro scale fluidic networks can dramatically simplify the screening process, providing a compartmentalized platform for nanoliter aliquots.

\section{General considerations}

During the past years, microfluidics, micrometer-scale total analysis systems, ( $\mu$ TAS) or so-called 'lab-on-a-chip (LOC)' devices have revised interest in the scaling laws and dimensionless groups for downscaling purposes [6]. The term 'microfluidic' refers to the ability to manipulate fluids in one or more channels with dimensions of 5-500 $\mu \mathrm{m}$ [7]. The development of microfluidics already started twenty years ago in inkjet printer manufacturing. The ink jet mechanism involves very small tubes carrying the ink. Etching technologies adapted from semiconductor technology are employed to create channels, tubes and chambers in silicon or glass substrates, which can be layered on top of one another to result in more complex 2-D and 3-D structures. Chip designs can also be stamped, molded or cut into plastic sheets. While the earliest reported microscale devices consisted of channels etched in solid substrates such as silicon [8], glass [9] and plastic [10], micro-electro-mechanical systems (MEMS), fabrication technologies have been increasingly applied to fabricate highly sophisticated devices from a variety of materials, including soft elastomers such as polydimethylsioxane (PDMS) [11] with hundreds of micro channels and integrated sensors to measure physiological parameters.

For the actuation of liquids on a solid surface, different approaches have been published so far [12]. Some pumping units are not an integral part of the chip and must be linked with appropriate tubes or pipes. They use, e.g., piezoelectric actuation or mechanically moving parts to drive the reagents through the channels. Others take advantage of the small dimensions of the microfluidic channel itself [13]. As the chemical potentials of the channel walls and the liquid inside may differ considerably, a space charge region forms at the interface. A voltage applied along the channel induces a flow within this space charge region, dragging along the liquid closer to the center of the channel.

*Corresponding author: Stefan Thalhammer, Helmholtz Zentrum München, Institute of Radiation Protection, Ingolstädterlandstrasse, 85764 Neuherberg, Germany, Tel: 49-89-3187-2893; Fax: 49-89-3187-3323; E-mail: stefan.thalhammer@helmholtz-muenchen.de

Received March 30, 2013; Accepted May 08, 2013; Published May 10, 2013

Citation: Thalhammer S, Wixforth A (2013) Surface Acoustic Wave Actuated Lab-on-Chip System for Single Cell Analysis. J Biosens Bioelectron 4: $13 \square$. doi:10.4172/2155-6210.100013

Copyright: (C) 2013 Thalhammer S, et al. This is an open-access article distributed under the terms of the Creative Commons Attribution License, which permits unrestricted use, distribution, and reproduction in any medium, provided the original author and source are credited. 
This electro kinetic effect works efficiently only for narrow channels and relatively high voltages. Fluidic motion can also be induced by spatially modulating the wetting properties of a substrate. For aqueous solutions, this can be achieved by, e.g., patterning the substrate with hydrophobic and hydrophilic regions. The techniques used to realize such a modulation of the wetting properties include micro-contact printing [14], vapor deposition, and photolithography [15]. Aqueous solutions prefer to cover the hydrophilic regions and avoid residing thereon. A guided flow can be achieved [16] by changing the wetting properties over time. For example, illumination can induce a motion of liquids as the free energy of the illuminated surface changes locally [17]. Other smart pumping mechanisms include peristaltic pumps based on thin membranes [18], or polymer films [19] with a controlled deformation creating a guided flow along microchannels [20]. More than 10.000 papers have been published over the last 10 years on the topic of microfluidics but no gold standard for actuation is presented so far. A critical review on LOC requirements, characteristics and applications is, e.g., given by the Zengerle group [21].

\section{Surface acoustic wave}

A surface acoustic wave (SAW) is an acoustic oscillation propagating at the surface of a solid. The SAW propagating parameters like, e.g., the amplitude typically decays in an exponential fashion with depth. SAW were first described in 1887 by Lord Rayleigh, who in his classic paper [22] on earthquakes reported on the modes of propagation and predicted the properties of such waves. Named for their discoverer, a special polarization mode of SAW, the 'Rayleigh wave' has a longitudinal and a vertical shear component which can couple to a medium in contact with the device's surface. SAW are especially easy to excite on piezoelectric substrates, where metal transducers on the surface convert an RF electrical signal into the desired mechanical oscillation. Coupling to any medium contacting the surface strongly affects the velocity or the amplitude of the wave [23]. As SAWs are confined to a thin layer at the substrate surface, their propagation crucially depends on the boundary conditions at this surface. Both mechanical as well as electrical load of the substrate surface significantly influences their propagation. Making use of this fact SAW devices can also be employed as sensor elements or to measure the dynamic conductivity of thin film devices [24].

\section{Surface acoustic wave based biosensors}

A biosensor, in principle, comprises a biochemical recognition system and a transducer, transforming the biochemical or biological response into a measureable signal [25]. The velocity of a SAW is sensitive to changes in the mass load of the active area, the viscosity of the material covering the active sensing area and the temperature of the surface $[23,26]$. Atypical SAW based sensor consists of a delay-line, assembled by two inter digital transducers (IDT) acting as an electrode pair on the piezoelectric substrate. The IDTs translate an electrical signal into a polarized transversal wave travelling parallel to the sensing surface. The amplitude and phase of the transmitted SAW with respect to the incoming one is detected by a second IDT. The differences in amplitude and phase are connected to changes in the mass load on the sensing area, viscosity, temperature or even conductivity on the surface of the sensing substrate [27]. With increasing frequency between 25 and $500 \mathrm{MHz}$, the penetration depth decreases, making the changes in the surface boundary conditions more important. Considering this effect, it turns out that the sensitivity of SAW sensors increases with frequency, yielding a sensor highly sensitive for surface interactions as most binding events occur in close proximity to the biosensor surface. Based on this, and as an example, a dual-delay-line a protein sensor to detect immunoglobulin $\mathrm{G}$ concentration in the range of 0.7 to $667 \mathrm{nM}$ was demonstrated [28]. Another approach highlighting the potential as a new diagnostic tool, a SAWimmuno-sensor to detect antigen-antibody binding with a sensitivity of $6 \mathrm{pg} / \mathrm{mm}^{2}$ was shown by Welsch and colleagues [29]. As biosensing applications very often rely on a detector in fluid environments, the out-of-plane elongation of a Rayleigh wave is not very convenient as it would lead to an undesired SAW attenuation just by viscous losses. Hence, as hear SAW, representing is a horizontally polarized shear wave is better suited. It has a high sensitivity for mass loading and (di) electrical changes of the surface environment by still maintaining the ability to operate in liquids with minimal propagation loss [26]. The technology could also be applied to whole cell analysis mainly focused on bacteria, bacteriophages and yeast as it was, e.g., used to detect $E$. coli bacteria to a threshold of $10^{6}$ bacteria/ $1 \mathrm{ml}$ in 500 $\mathrm{ml}$ test solution [30]. A comprehensive review of various types of SAW based sensors and applications can be found in [31].

\section{SAW sample processing}

Apart from the described sensing abilities, SAW can also be used as asource of energy and consequent heating in synthetic chemistry or protein workflows. Perlmutter and coworkers, for example, presented a droplet based micro reactor for synthetic chemistry [32]. By applying SAW with frequencies ranging from $1 \mathrm{Mhz}$ to more than $2 \mathrm{GHz}$ they could perform chemical reactions like Diels-Alder, Kabachnik-Foelds or Baylis-Hillman reaction. These reactions were carried out very fast and with high yields and clean products. Also, SAW based assembly and accumulation of proteins on supported lipid bilayers was shown [33] before. Here, homogenous mixtures of proteins of different size are separated into individual fractions and accumulated reversibly into spatially and temporally controlled pattern by SAW. SAWs can also be used for protein in-gel processing. This was demonstrated by Perlmutter and coworkers by using SAW to prepare, rehydrate, in situ digest and extract peptides from gel slices [34]. The results showed similar coverage and intensity in base peak chromatograms like traditional processing. The excellent peptide recoveries, rapid turnover of sample and high confidence protein identifications makes them a powerful new approach to the manipulation and processing of chemicals and peptides.

\section{Actuation by surface acoustic waves}

In most conventional microfluidic systems, liquids are confined and actuated in closed tubes, trenches or capillaries. Usually, the application of such systems is restricted to continuous flow processes. To realize the concept of microfluidics in such closed system, some major problems need to be overcome. For example, the pressure required for actuating the liquid scales with the channel dimension [35]. Furthermore, shrinking of liquid handling systems down to the micron and submicron size range unavoidably leads into the regime of small Reynolds numbers. The fluid dynamics in this regime are very different from the macroscale. For example, we are used to seeing liquids mix by turbulent flow, as illustrated by the way cream swirls into coffee. But such turbulence does not occur in closed channel or tubing systems just a few micrometers wide. They are governed by the rules of laminar flow or low Reynold's numbers [36]. The Reynolds number $(\mathrm{Re})$ is a dimensionless quantity describing the ratio between viscosity and inertia related effects and is defined as

$$
\operatorname{Re}=v l p / \eta
$$

Here, $v$ is the fluid velocity, $l$ is the characteristic channel 
dimension, $\rho$ is the fluid density and $\eta$ is the fluid viscosity. At high Reynolds numbers (that is, $\operatorname{Re}>2000$ ), fluid flow can be turbulent. At low Reynolds numbers (that is, $\operatorname{Re}<2000$ ), fluid flow is mostly laminar.

In micro devices with channel diameter smaller than $1 \mu \mathrm{m}$, the flow is usually governed by very low Reynolds number conditions $(\operatorname{Re}<1)$, where turbulence and inertia effects are basically non-existent. Although the theoretical description and modelling is simpler in this case (the nonlinear Navier Stokes equation under these conditions is replaced by the much simpler Stokes equation) mixing, for example, is typically only driven and dominated by diffusion. Hence, under these conditions diffusion can no longer be neglected for the design of these devices. Moreover, forces due to surface tension at liquid/air interfaces become dominant and gravity or inertia related effects are totally negligible. As the magnitude of the physical effects in the microfluidic world are very much different from those at macroscopic scale, fluid-integrated micro devices must be designed from first principles, rather than simply by miniaturizing macroscopic devices. As a result of this, the power of the employed pumping units has to be typically increased while reducing the size, which complicates the integration into a complete system. Furthermore, when a biological solution is pumped through a narrow tube, the risk of reagent loss by adhesion to the wall is large due to the unfavorable surface to volume ratio. Other problems arise by small channels becoming easily clogged and by surface modification and functionalization being difficult to control.

A smart alternative to pumping and electro kinetic principles to transport droplets along the surface is surface acoustic wave actuation with amplitudes of typical only a few nanometers [24]. The driving force behind this interaction and the resulting acoustically driven flow is an effect called 'acoustic streaming [20]. It is a consequence of the pressure dependence of the mass density, leading to a non-vanishing time average of the acoustically induced pressure. Although acoustic streaming has been a well-known effect for a long time in macroscopic, classical systems, little attention has been paid to it, so far, in terms of miniaturization [37].

The basic mechanism for acoustic streaming is depicted in figure 1a. Here, a SAW carrying substrate is shown, partially covered by a liquid droplet to the right. The propagating SAW couples to a sound wave in the liquid. As the sound velocity in fluids $\mathrm{v}_{\text {liquid }}$ is smaller than the SAW velocity in the solid substrate, sound is radiated into the liquid under an angle obeying Snellius' law of diffraction, the so-called Rayleigh angle $\Theta_{\mathrm{R}}$ :

$$
\Theta_{R}=\arcsin \frac{v_{\text {liqiuid }}}{v_{S A W}}
$$

Anacoustic wave entering the droplet is diffracted under this Rayleigh angle $\Theta_{R}$ into the fluid, where it generates a longitudinal pressure wave. For an infinite half-space, the diffraction angle is only given by the ratio of the sound velocities in the substrate and in the fluid, respectively, and can be understood as a consequence of phase matching between the SAW and the radiated sound beam in the liquid. Sound travelling through a liquid is viscously attenuated along its transmission through the medium. If the intensity is high, this attenuation creates an acoustic pressure gradient along the propagation of the wave. The gradient induces a force in the same direction, which in turn causes a flow in the fluid. Such 'mode conversion' leads to an exponential decay of the SAW along its path on the chip. Experimentally, in this case one typically notices a viscous attenuation of about $1 \mathrm{dBm}$ per wavelength [38].

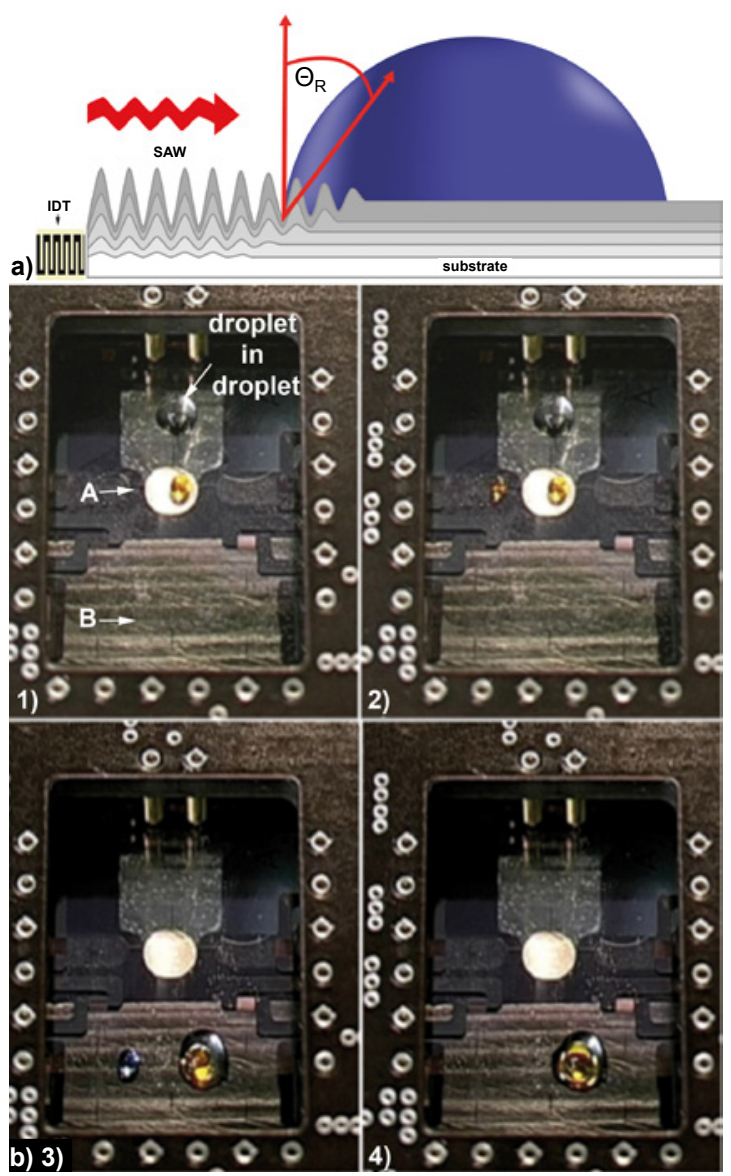

Figure 1: a) A schematic illustration of the interaction between a surface acoustic wave (SAW) and a fluidat the surface of the $\mathrm{LiNbO} 3$ substrate. The SAW is propagating from left to right, impinging the liquid at $x=0$. A longitudinal sound wave is radiated into the fluid under a refraction angle $\Theta R$. b) The SAW driven microfluidic lab-on-a-chip. Four droplets $(\approx 100 \mathrm{nl}$ each) and a $5 \mu \mathrm{l}$ cover droplet of mineral oil are moved in a "remotely controlled" manner and independently by the nanopumps; 1) through 4) represent a series of snapshots of the lab-on-a-chip system to visualize the movement and the "nanochemical" reactions occurring when the droplets are merged and mixed in the reaction centers $A$ and $B$ by the action of the surface wave.

After the first successful implementation of the SAW streaming at surfaces [24] some other groups also picked up on this research. For example, very effective actuation of small amounts of liquids in the range from $2 \mathrm{nl}$ to $20 \mu \mathrm{l}$ and propelling a water droplet at $40 \mathrm{~mm} / \mathrm{sec}$ was shown [39]. Moreover, two or more droplets can be merged the mixture of these substances is quickly released compared to diffusion processes. This is due to the internal mixture in the droplet caused by SAW streaming [24]. Furthermore, these acoustic waves in a solid lead to pronounced streaming effects in the fluid inducing mixing and stirring even at low Reynold's numbers [40].

The mode of operation of such SAW driven microfluidic applications determines their actual use. SAW can either be excited continuously or pulsed, at low power levels or at high ones. Two opposing SAWs may interfere into a standing wave, where no net flow of the fluid is observed but still a very active internal flow (mixing) is generated. Perlmutter [32], for example, employed the SAW induced temperature increase to trigger drop based chemical reactions. Droplet actuation in our system is usually performed in a pulsed mode of operation; 

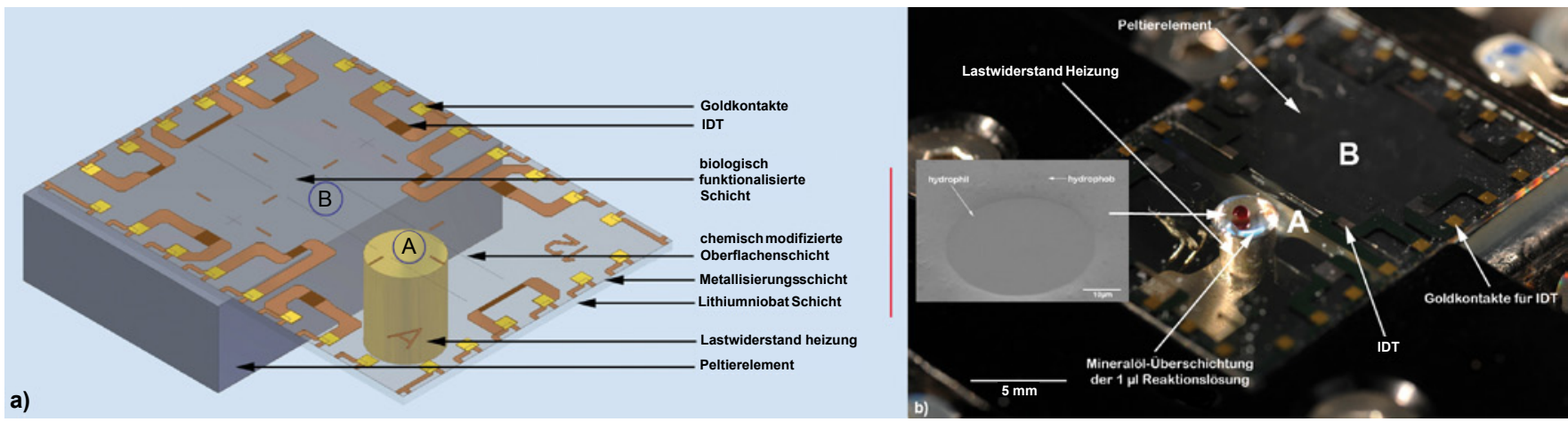

Figure 2: a) Sketch of LOC functionality. The host substrate (LiNbO3) is covered by a laterally patterned layer of Pt, Ni and Au for transducers and sensor metallization. Subsequent silanisation of the surface accounts for a hydrophilic/hydrophobic surface chemistry, facilitating a planar fluid track system, which could be further functionalized by a problem-specific detection array. Reaction center A is controlled by a load resistor heating, reaction point $B$ by a peltier element b) optical image of the chip installed in the heating unit; on reaction center A a $1 \mu \mathrm{l}$ aqueous solution is covered by $5 \mu \mathrm{l}$ mineral oil; scale bar $5 \mathrm{~mm}$. Inset: electron microscope images of the hydrophilic reaction center $A$ on the hydrophobic chip surface, scale bar $10 \mu \mathrm{m}$.

a)
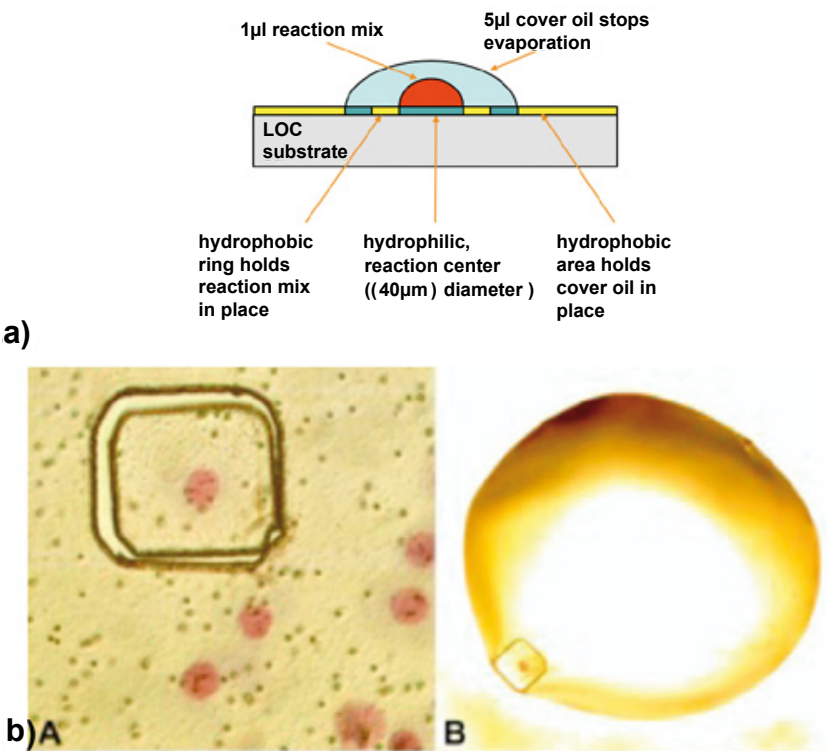

Figure 3: a) Microdissection and particle transfer via SPATS, integrated into a conventional inverted optical microscope for single particle transfer to the Lab-on-a-Chip (LOC): micrometer step motor providing exact positioning, the carrier trunk with easy-to-fit click system, the sample adsorbing head and the collection grid centered to a sample slide; b) workflow of the single particle isolation using SPATS43:

A) a single cell $(\sim 20 \mu \mathrm{m})$ is isolated and precisely released into

B) a $200 \mathrm{nl}$ droplet of buffer solution;

C) sketch of the planar hydrophilic/hydrophobic structured glass slide with $1 \mu \mathrm{l}$ PCR reaction mixture covered with $5 \mu$ mineral oil.

where due to the short pulses and the high thermal conductivity of the substrate temperature related effects may be basically neglected. Hence, the (computer controlled) mode of operation offers yet many other tuning knobs without the need for additional hardware for basically any desired application. This is especially true, if the LOC apart from the SAW related infrastructure also hosts 'conventional' functional units like heaters, thermometers and alike.

\section{SAW Driven Freely Programmable Lab-on-a-chip System}

Instead of closed tubing, a chemically modified surface providing droplet based virtual fluid confinements was developed (Figure 2b).
The shape of a droplet on a surface is given by the properties of the substrate and is controlled by the surface free energy and not by channel walls. Depending on the chemical modification, droplets with high contact angle will form (hydrophobic) or the liquid will preferentially wet the surface (hydrophilic). They form their own 'virtual' test tubes, held together by surface tension effects. The agitating surface acoustic waves are generated by high frequency electrical signals on micro structured inter digital transducers embedded into the lab-on-a-chip. The upper boundary of a real, spatially confined fluid, defined by its surface tension bends the streaming lines, resulting in a continuous flow within the droplet [38]. At larger SAW amplitudes, the internal streaming develops into a movement of the droplet as a whole into the desired direction on the chip. The technology to create such fluidic tracks very much resembles the one used to define conducting paths on an electronic semiconductor device. A true lab-on-a-chip, however, requires more than just test tubes. More importantly, their cargo has to be moved around, mixed, stirred or processed in general by combing laminar and turbulent flow in $5 \mu \mathrm{l}$ droplets by changing the surface properties via chemical lithography. This approach is also sometimes referred to as "flat fluidics" [21,41].

Probably one of the first complex SAW based labs on a chip was a system for multi-spot PCR [42]. Here, the chip not only contained the SAW actuation rapid mixing but also heaters and thermometers for a successful DNA amplification on a chip. Very intriguingly, the SAW mixing in this case could also be employed to dissolve the necessary chemicals, which were spotted on the chip before actual use in dried form.

In more complex systems and in contrast to conventional closed microfluidic systems with external pumping units, SAW are employed to agitate and actuate these little virtual test tubes along predetermined trajectories [24]. The interaction between the SAW and the fluid on the chip leads to acoustic streaming within the fluid, which apart from actuation and mixing within a closed volume can also convey the small droplets as a whole. Liquid volumes in the range from 1 micro- down to 100 picoliterare in this case precisely moved on pre defined fluidic 'tracks'. The reagents are manipulated either as discrete droplets or by streaming patterns induced in somewhat larger volumes. Velocities close to $1 \mathrm{~m} / \mathrm{sec}$ can be achieved in this way. As the SAW nano pumps are electrically addressable, a complete sequence of different steps of biochemical reactions or biological assays can be computer controlled. Moreover, the simplicity of the fabrication process of the freely 
Citation: Thalhammer S, Wixforth A (2013) Surface Acoustic Wave Actuated Lab-on-Chip System for Single Cell Analysis. J Biosens Bioelectron 4: 13! . doi:10.4172/2155-6210.100013!

1)

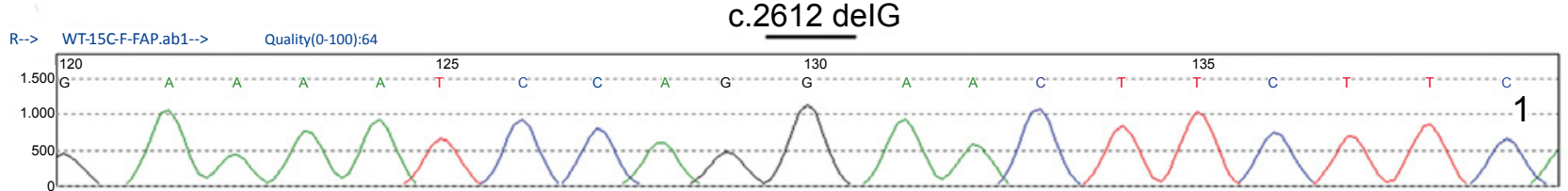

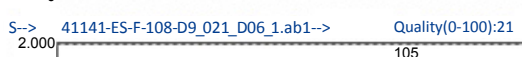

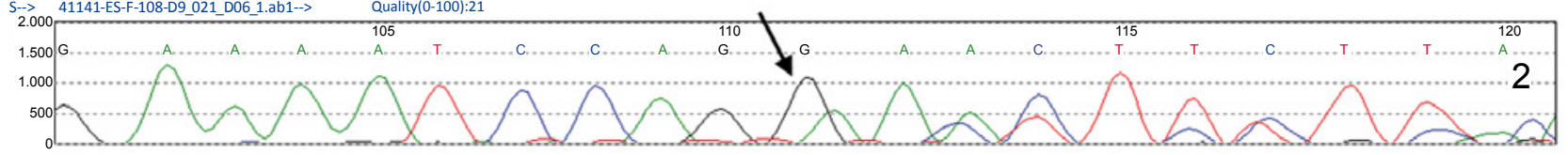

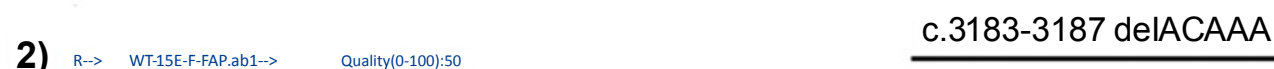

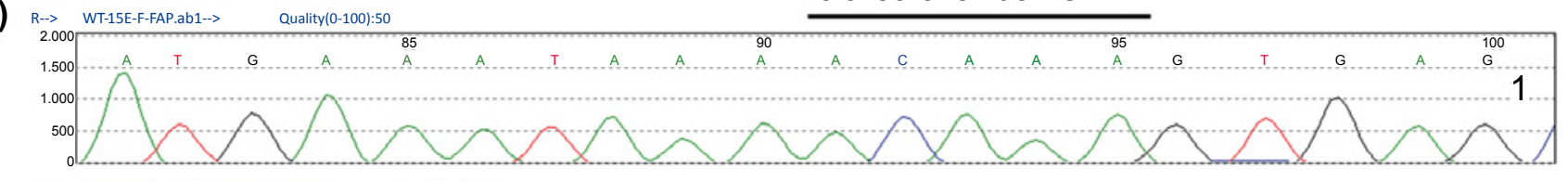

S--> 47031-136-Al-F-C6_G05_013_1.ab1-> Quality(0-100):0

a)

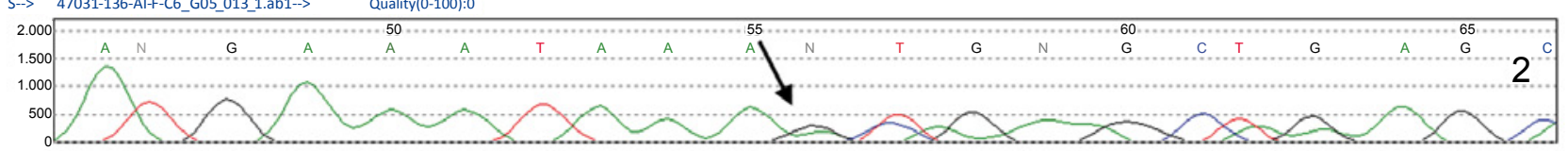

b)

1)
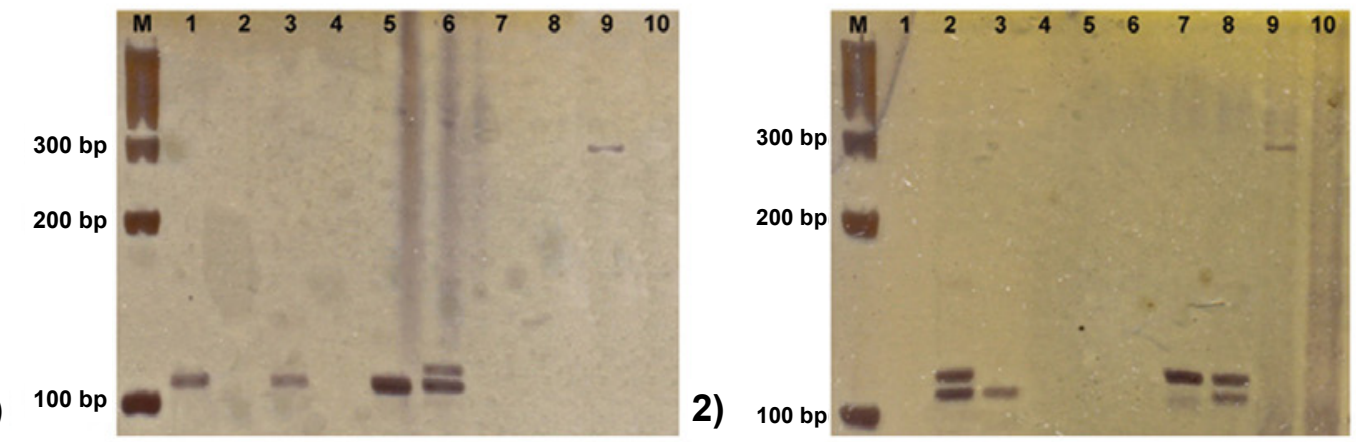

Figure 4: a) Specific mutations in the APC gene of FAP.

A) Sequencing shows the heterozygous 1 bp deletion (c.2612delG) (arrow) of case1. Lane 1: wild type sequence of exon 15; lane 2: forward case specific sequence B) shows the wild type sequence in lane 3 and the heterozygous sequence (forward) with the 5 bp deletion (c.3183-3187delACAAA) (arrow) of case1 in lane 4 (@S. Karger AG, Basel, Switzerland); b) Polyacrylamid gel electrophoresis of amplified mummy DNA. PCR was performed on fragments of the human gene $\beta$-actin (297 $\mathrm{bp}$ ) and the sex specific amelogenin gene (female 106bp (sample 1)), male 106/112 bp (sample 2), lane description: M: $100 \mathrm{bp}$ molecular length standard (Peqlab, Germany): 1) lane 1: Amelogenin PCR (SPATS DNA extraction method) of 60 pg female mummy DNA;lanes 2, 7, 4, 8: Amelogenin PCR (supporting membrane, lysis buffer, PCR master mix and $\mathrm{H}_{2} \mathrm{O}$ control as negative controls); lane 3: Amelogenin PCR (conventional DNA extraction method) 50 pg female mummy DNA; lane 5+6: Amelogenin PCR (100 pg male and female human reference DNA, positive control); lane 9: $\beta$-actin PCR of $\sim 60$ pg mummy DNA (SPATS DNA extraction method); lane 10: $\beta$-actin PCR negative control (PCR master mix). 2) lanes 1, 4, 5, 6: Amelogenin PCR (supporting membrane, lysis buffer, PCR master mix and $\mathrm{H}_{2} \mathrm{O}$ control as negative controls); lane 2+3: Amelogenin PCR (100 pg male and female human reference DNA, positive control); lane 7, Amelogenin PCR (conventional DNA extraction method) 100pg male mummy DNA; lane 8: Amelogenin PCR (SPATS DNA extraction method) of $\sim 60$ pg male; lane 9: $\beta$-actin PCR of $\sim 60$ pg mummy DNA (SPATS DNA extraction method); lane 10: $\beta$-actin PCR negative control (PCR master mix) (@ John Wiley \& Sons, Ltd).

programmable "bio processor" in combination with the possibility to operate and control the entire process via computer automation, makes the developed system an ideal candidate for question- and patientspecific truly miniaturized laboratories on a chip (Figure 1b).

In this way, well-defined analyses, controlled in the sub-microlitre regime, can be quickly and gently conducted on such an acoustically driven lab-on-a-chip. Apart from its nearly unlimited applicability for many different biological assays, its programmability and extremely low manufacturing costs are another definite advantage of the "freely programmable lab-on-a-chip" [43] (Figure 2a). The technology allows for both batch and continuous processes to be carried out at high speed. In fact, they can be produced at such low cost that their use as disposables in many areas of diagnosis can be envisioned. The most important feature, however, is the programmability of the chip as different assay protocols can be realized with the same chip layout and hardware.

\section{Molecular Biology}

The analysis of genetic material is fundamental to various medical and scientific applications. These include diagnosis of genetic disorders from prenatal diagnosis, investigation of oncogenes, forensic analysis, disease diagnosis and screening, paternity testing, gene therapy formation and detection of pathological specimen. But the primary reason for the analysis of genetic material, particularly in medicine, is the diagnosis of genetic disorders. Reducing PCR to the microliter level (Figure 3a) is not only of interest for portable detection technologies and 
high-throughput and parallel analytical systems. Also, as a consequent next step towards an individualized therapy or point of care diagnosis (POC) it is essential to dissipate the complexity in heterogeneous tissue sections.

To load the fluidic chip with the substances of interest, minute amounts of sample material are extracted by laser-based micro dissection out of e.g. histological sections [44]. A few picogram of genetic material are thus isolated and transferred via a low-pressure transfer system [45] onto the chip (Figure 3b). This even allows for the processing of living biological samples on the single cell level and within these 'biological reactors' the genetic material is finally processed, e.g. amplified via PCR methods [42]. The highest sensitivity that can be achieved with a genetic analysis device is the successful amplification of a single DNA template and the subsequent detection of single nucleotide alterations. As feasibility study for our LOC by is for instance a single cell assay: We have chosen this application example as it addresses most relevant questions in molecular analysis, dealing with minimum target amount of DNA, to avoid background noise of unwanted DNA, contamination risks and detection limits. By using this droplet-in-droplet configuration, single DNA base pair deletion could be detected after single cell isolation and processing [46]. When only a minute amount of DNA is available, the simultaneous amplification of polymorphic marker sequences combined with the amplification of the mutated gene region is important in order to avoid misdiagnosis due to preferential amplification, allelic drop out or contamination. For the analyzed $1 \mathrm{bp}$ deletion, the results of the sequencing analysis were confirmed using the Snapshot approach, which allows reliable genotyping of the mutation (Figure $4 \mathrm{a}$ ). All major results of the single cell analysis are for example summarized in [46].

\section{Forensic Sciences}

However, despite the addressed limiting problems of PCR towards a parallelized high throughput analysis, the majority of subsequent problems are caused by a series of steps in sample preparation and the isolation of purified nucleic acid templates. As an ambitious challenge, we here performed sex determination on ancient bone material: The study of ancient DNA (aDNA) plays a significant role in archaeological and paleontological research as well as in pathology and forensics. Various aDNA extraction methods are currently in use, which rely on different principles like spin column, alcohol precipitation or silica binding. All of these methods aim to maximize DNA yields, while minimizing the co-extraction of PCR inhibitors. While working with a DNA, originating and extracted from excavated bones and teeth, external inhibiting factors problems like low DNA quantity, high DNA degradation, DNA contamination and the presence of PCR inhibitors can reduce the PCR quality and hence the results [47]. There are numerous precautions that have to be taken in order to minimize the hazard of amplifying contaminating modern DNA in ancient specimens [48]. Our LOC addresses the specified problems and can circumvent these according to a) minimization of contamination during handling of specimens, b) sample extraction exclusive from internal bone parts with a minimization of degrading effects and co-extraction of inhibiting substances and c) a reduction in the amount of starting material down to single osteon islets allowing maximum preservation of ancient material [49]. DNA amplification experiments were performed using low-volume PCR following the droplet-in-droplet configuration in $1 \mu \mathrm{l}$ total reaction volume covered with $5 \mu$ l of mineral oil (Figure 4b). Amplification was performed on 106/112 bp fragments of the amelogenin gene for gender determination, as well as on a larger fragment of the human $\beta$-actin gene comprising $297 \mathrm{bp}$. Amplified gene fragments were rated as reproducible and authentic only after a) five PCR reactions showed consistent fragment determination, $b$ ) this result could be reproduced in another extract from the same sample and c) all controls were negative. The reliability of this method was as high as $90-95 \%$.Sequencing analysis was performed with amelogenin and $\beta$-actin PCR products, amplified from a male mummy sample. With respect to extract a DNA quality, the grade of DNA degradation, and interfering destructive factors, our approach significantly outperformed conventional tube based methods. Analysis of the sequencing data for both the 106/112 bp X- and Y-chromosomal amelogenin fragments and the $297 \mathrm{bp} \beta$-actin fragment definitively identified them as human amelogenin and $\beta$-actin sequences once aligned to gene sequences from the human genomic database of NCBI [49].

\section{Conclusion}

Our SAW driven, freely programmable lab-on-a-chip system allows for the isolation and transfer of smallest amounts of genetic material on a micro scale precision. On the planar and chemically patterned substrate, droplets are contactless actuated via surface acoustic waves and are confined in virtual reaction chambers for biochemical processing. Compared to PCR analysis in larger reaction volumes, this leads to an increased amplification sensitivity and efficiency of PCR, which is necessary for minute amounts of DNA. This technique allows for a variety of molecular analyses where isolation of few or single cells is important for genetic characterization, e.g. heterogeneous cell populations or mosaics. Even the isolation and analysis of specific regions from tissue sections (e. g. tumor sections) is facilitated with our system. Due to the selective isolation and single particle transfer processes, cross-contamination with tissue cells of neighboring regions can be circumvented.

Apart from time saving and cost reduction, our system combines highest sensitivity and reliability towards a fully automated lab-on-achip in the increasing field of point-of-care diagnosis and individualized therapies.

\section{Acknowledgement}

Generous financial support by the Bavarian Research Foundation under contract number AZ-669-05 is gratefully acknowledged.

\section{References}

1. Andersson $\mathrm{H}$, van den Berg A (2004) Microtechnologies and nanotechnologies for single-cell analysis. Curr Opin Biotechnol 15: 44-49.

2. Vickerman V, Blundo J, Chung S, Kamm R (2008) Design, fabrication and implementation of a novel multi-parameter control microfluidic platform for three-dimensional cell culture and real-time imaging. Lab Chip 8: 1468-1477.

3. Kricka LJ, Wilding P (2003) Microchip PCR. Anal Bioanal Chem 377: 820-825.

4. Wang J (2002) Portable electrochemical systems. Trends Anal. Chem 21: 226 232

5. Verpoorte E (2002) Microfluidic chips for clinical and forensic analysis Electrophoresis 23: 677-712.

6. Manz A, Graber N, Widmer HM (1990) Miniaturized total chemical analyses systems. A novel concept for chemical sensing. Sens Actuators B Chem 1 244-248.

7. Weibel DB, Whitesides GM (2006) Applications of microfluidics in chemical biology. Curr Opin Chem Biol 10: 584-591.

8. Manz A, Miyahara Y, Miura J, Watanabe Y, Miyagi H, et al. (1990) Design of an open-tubular column liquid chromatography using silicon chip technology. Sens Actuators B Chem 1: 249-255

9. Harrison DJ, Fluri K, Seiler K, Fan Z, Effenhauser CS, et al. (1993) Micromachining a miniaturized capillary electrophoresis-based chemical analysis system on a chip. Science 261: 895-897. 
Citation: Thalhammer S, Wixforth A (2013) Surface Acoustic Wave Actuated Lab-on-Chip System for Single Cell Analysis. J Biosens Bioelectron 4: 13!. doi:10.4172/2155-6210.100013[

10. Martynova L, Locascio LE, Gaitan M, Kramer GW, Christensen RG, et al. (1997) Fabrication of plastic microfluid channels by imprinting methods. Ana Chem 69: 4783-4789.

11. Duffy DC, McDonald JC, Schueller OJ, Whitesides GM (1998) Rapid Prototyping of Microfluidic Systems in Poly(dimethylsiloxane). Anal Chem 70: 4974-4984.

12. Li PCH (2006) Chromatographic science series 94, Taylor \& Francis CRC Press.

13. Harrison DJ, Fluri K, Seiler K, Fan Z, Effenhauser CS, et al. (1993) Micromachining a miniaturized capillary electrophoresis-based chemical analysis system on a chip. Science 261: 895-897.

14. López GP, Biebuyck HA, Frisbie CD, Whitesides GM (1993) Imaging of features on surfaces by condensation figures. Science 260: 647-649.

15. Gau H, Herminghaus S, Lenz P, Lipowsky R (1999) Liquid morphologies on structured surfaces: from microchannels to microchips Science 283: 46-49.

16. Daniel S, Chaudhury MK, Chen JC (2001) Fast drop movements resulting from the phase change on a gradient surface. Science 291: 633-636.

17. Ichimura K, Oh SK, Nakagawa M (2000) Light-driven motion of liquids on a photoresponsive surface Science 288: 1624-1626.

18. Moroney RM, White RM, Howe RT (1991) Microtransport induced by ultrasonic Lamb waves. Appl Phys Lett 59: 774-776.

19. Unger MA, Chou HP, Thorsen T, Scherer A, Quake SR (2000) Monolithic microfabricated valves and pumps by multilayer soft lithography. Science 288 113-116.

20. Nyborg WL (1965) Acoustic Streaming, in Physical Acoustics 2B 265, Ed. W.P. Mason, Academic Press.

21. Mark D, Haeberle S, Roth G, von Stetten F, Zengerle R (2010) Microfluidic labon-a-chip platforms: requirements, characteristics and applications. Chem Soc Rev 39: 1153-1182.

22. Lord Rayleigh OMFRS (1905) On the momentum and pressure of gaseous vibrations, and on the connexion with the virial theorem. Philosophical Magazine Series 6: 364-374.

23. Gronewold TM (2007) Surface acoustic wave sensors in the bioanalytical field: recent trends and challenges. Anal Chim Acta 603: 119-128.

24. Wixforth A (2003) Acoustically driven planar microfluidics. Superlattices and Microstructures 33: 389-396.

25. Thévenot DR, Toth K, Durst RA, Wilson GS (1999) Electro chemical biosensors: recommended definitions and classification. Pure Appl Chem 71: 2333-2348.

26. Ballantine DS (1997) Acoustic wave sensors: Theory, Design, and physicochemical apllications. Academic Press CA USA.

27. Arruda DL, Wilson WC, Nguyen C, Yao QW, Caiazzo RJ, et al. (2009) Microelectrical sensors as emerging platforms for protein biomarker detection in point-of-care diagnostics. Expert Rev Mol Diagn 9: 749-755.

28. Gizeli E, Bender F, Rasmusson A, Saha K, Josse F, et al. (2003) Sensitivity of the acoustic waveguide biosensor to protein binding as a function of the waveguide properties. Biosens Bioelectron 18: 1399-1406.

29. Weisch W, Klein C, von Schickfus M, Hunklinger S (1996) Development of a surface acoustic wave immunosensor. Anal Chem 68: 2000-2004.

30. Moll N, Pascal E, Dinh DH, Pillot JP, Bennetau B, et al. (2007) A Love wave immunosensor for whole E. coli bacteria detection using an innovative two-step immobilisation approach. Biosens Bioelectron 22: 2145-2150.

31. Länge K, Rapp BE, Rapp M (2008) Surface acoustic wave biosensors: a review. Anal Bioanal Chem 391: 1509-1519.

32. Kulkarni K, Friend J, Yeo L, Perlmutter P (2009) Surface acoustic waves as an energy source for drop scale synthetic chemistry. Lab Chip 9: 754-755.
33. Neumann J, Hennig M, Wixforth A, Manus S, Rädler JO et al. (2010) Transport, separation, and accumulation of proteins on supported lipid bilayers. Nano Let 10: $2903-2908$.

34. Kulkarni KP, Ramarathinam SH, Friend J, Yeo L, Purcell AW, et al. (2010) Rapid microscale in-gel processing and digestion of proteins using surface acoustic waves. Lab Chip 10: 1518-1520.

35. Brody JP, Yager P, Goldstein RE, Austin RH (1996) Biotechnology at low Reynolds numbers. Biophys J 71: 3430-3441.

36. Barry NP, Bretscher MS (2010) Dictyostelium amoebae and neutrophils can swim. Proc Natl Acad Sci U S A 107: 11376-11380.

37. Eckart C (1947) Vortices and streams caused by sound waves. Phys Rev 73 68-76.

38. Wixforth A (2000) Interaction of surface acoustic waves, electrons, and light. Int J High Spe Ele Syst 10: 1193-1227.

39. Beyssen D, Le Brizoual L, Elmazria O, Alnot P (2006) Microfluidic device based on surface acoustic wave. Sens Actuators B 118: 380-385.

40. Sritharan K, Strobl CJ, Schneider MF, Wixforth A, Guttenberg Z (2006) Acoustic mixing at low Reynold's numbers. APL 88: 054102.

41. Wixforth A, Gauer C, Scriba J, Wassermeier M, Kirchner R (2003) Flat fluidics: A new route toward programmable biochips. Proceedings of SPIE 4982: 235 242.

42. Guttenberg Z, Muller H, Habermüller H, Geisbauer A, Pipper J, et al. (2005) Planar chip device for PCR and hybridization with surface acoustic wave pump. Lab Chip 5: 308-317.

43. Thalhammer S (2009) Programmable Lab-on-a-Chip system for single cell analysis. Proc SPIE 7364: 73640B.

44. Thalhammer S, Lahr G, Clement-Sengewald A, Heckl WM, Burgemeister R, et al. (2003) Laser microtools in cell biology and molecular medicine. Laser Physics 13: 681-691.

45. Woide D, Mayer V, Wachtmeister T, Hoehn N, Zink A, et al. (2009) Single particle adsorbing transfer system. Biomed Microdevices 11: 609-614.

46. Mayer V, Schoen U, Holinski-Feder E, Koehler U, Thalhammer S (2009) Single cell analysis of mutations in the APC gene. Fetal Diagn Ther 26: 148-156.

47. Primorac D, Andelinovic S, Definis-Gojanovic M, Drmic I, Rezic B, et al (1996) Identification of war victims from mass graves in Croatia, Bosnia, and Herzegovina by use of standard forensic methods and DNA typing. J Forensic Sci 41: 891-894.

48. Pääbo S, Poinar H, Serre D, Jaenicke-Despres V, Hebler J, et al. (2004) Genetic analyses from ancient DNA. Annu Rev Genet 38: 645-679.

49. Woide D, Zink A, Thalhammer S (2010) Technical note: PCR analysis of minimum target amount of ancient DNA. Am J Phys Anthropol 142: 321-327. 